\title{
Focused Ultrasound: An Emerging Therapeutic Modality for Neurologic Disease
}

\author{
Paul S. Fishman ${ }^{1} \cdot$ Victor Frenkel $^{2}$
}

Published online: 27 February 2017

(C) The American Society for Experimental NeuroTherapeutics, Inc. 2017

\begin{abstract}
Therapeutic ultrasound is only beginning to be applied to neurologic conditions, but the potential of this modality for a wide spectrum of brain applications is high. Engineering advances now allow sound waves to be targeted through the skull to a brain region selected with real time magnetic resonance imaging and thermography, using a commercial array of focused emitters. High intensities of sonic energy can create a coagulation lesion similar to that of older radiofrequency stereotactic methods, but without opening the skull. This has led to the recent Food and Drug Administration approval of focused ultrasound (FUS) thalamotomy for unilateral treatment of essential tremor. Clinical studies of stereotactic FUS for aspects of Parkinson's disease, chronic pain, and refractory psychiatric indications are underway, with promising results. Moderate-intensity FUS has the potential to safely open the blood-brain barrier for localized delivery of therapeutics, while low levels of sonic energy can be used as a form of neuromodulation.
\end{abstract}

Keywords Focused ultrasound · essential tremor .

Parkinson's disease · blood-brain barrier · neuromodulation · MRgFUS

Paul S. Fishman

pfishman@som.umaryland.edu

1 Department of Neurology, University of Maryland School of Medicine, Baltimore, MD 21201, USA

2 Department of Diagnostic Radiology and Nuclear Medicine, University of Maryland School of Medicine, Baltimore, MD 21201, USA

\section{Introduction}

The use of diagnostic ultrasound is a well-established method; however, there is only growing awareness of ultrasound as a potential therapeutic modality for neurologic disease. With the recent first Food and Drug Administration (FDA) approval (July 2016) of this novel treatment for a neurologic condition [essential tremor (ET)], both preclinical and clinical research are expanding rapidly for several neurologic indications. Much of this progress is a result of improving technology to provide controlled levels of ultrasonic energy that is focused to a brain target through the skull and guided by magnetic resonance imaging (MRI). The goals of therapeutic ultrasound of the brain can be broadly related to the level of ultrasound energy provided (Table 1). High-intensity focused ultrasound (HIFU) is sufficient to create a coagulation lesion in the brain with the goal of developing a substantially less invasive way to create stereotactic brain lesions. Moderate levels of focused ultrasound (FUS) energy can be employed to safely open the blood-brain barrier (BBB) for localized delivery of therapeutics. Relatively low levels of FUS can affect neural tissue and be used as a form of neuromodulation for both stimulation and suppression of neuronal activity.

\section{A Brief History of FUS}

The potential of focused ultrasound (FUS) as a viable treatment modality was first shown as early as the 1940 s, predating the use of ultrasound for imaging. In a number of experimental studies, these exposures were found to create localized bio-effects in the brain of preclinical models, identified by well-characterized modifications in behavior [13]. Not until recently, however, with the advent of state-of-the-art imageguided FUS devices, have clinicians begun to truly realize the 
Table 1 Summary of focused ultrasound (FUS) applications in the brain and underlying mechanisms

\begin{tabular}{|c|c|c|c|}
\hline FUS exposure & Effect & Mechanism & Application \\
\hline High intensity $(\mathrm{CW})$ & $\begin{array}{l}\text { Thermal; irreversible tissue } \\
\text { destruction }\end{array}$ & Coagulative necrosis & $\begin{array}{l}\text { Thalamotomy for ET [1], PD [2], } \\
\text { and neuropathic pain [3] }\end{array}$ \\
\hline Medium intensity $(\mathrm{PW})$ & $\begin{array}{l}\text { Mechanical; transient opening } \\
\text { of the BBB }\end{array}$ & $\begin{array}{l}\text { Activation/stable oscillation of UCA } \rightarrow \text { shear } \\
\text { stress and direct interactions }\end{array}$ & $\begin{array}{l}\text { Enhanced delivery of antitumor } \\
\text { agents }[4,5] \text {, gene therapy, } \\
\text { cells }[6-8]\end{array}$ \\
\hline Low intensity (PW) & $\begin{array}{l}\text { Mechanical; neuromodulation } \\
\text { (stimulation and suppression) }\end{array}$ & $\begin{array}{l}\text { Thought to be related to mechanical perturbation } \\
\text { of voltage-dependent ion channels or changes } \\
\text { in bilayer impedance }\end{array}$ & $\begin{array}{l}\text { Activation of motor response } \\
{[9,10], \text { suppression of VEP }} \\
{[11] \text { and acute epileptic }} \\
\text { activity [12] }\end{array}$ \\
\hline
\end{tabular}

$\mathrm{CW}=$ continuous wave; $\mathrm{ET}$ = essential tremor; $\mathrm{PD}=$ Parkinson's disease; $\mathrm{PW}=$ pulsed wave; $\mathrm{BBB}=$ blood-brain barrier; $\mathrm{UCA}=$ ultrasound contrast agents; $\mathrm{VEP}=$ ??

potential of this minimally invasive technology as a viable, faster, and safer alternative for the treatment of many diseases and disorders. Applications of image-guided FUS are diverse and depend on the manner in which the exposures are provided [14].

Interest and development in FUS technology, in general, and its clinical implementation continue to grow, where presently hundreds of research centers and universities worldwide are working to develop new applications, and improve existing ones. Clinically approved applications include lowintensity, nonfocused exposures for healing in physical therapy [15], and higher-intensity FUS for noninvasively ablating a variety of benign and malignant tumors [16]. The latter includes the treatment of uterine fibroids [17], breast cancer [18], and bone metastasis [19]. FUS is FDA approved for uterine fibroids, bone metastases, prostate cancer, and benign prostatic hyperplasia. FUS is approved for breast cancer outside of the USA and is currently in clinical trials in the USA. Thermal ablation of prostate tumors was the original oncological application of FUS treatment. A recent 10-year follow-up analysis showed FUS to be safe and effective as a whole-gland primary treatment for localized prostate tumors [20]. The advantages of FUS include the ability for repeated treatments with no cumulative effect, where mechanically registered imaging modalities can be used for both treatment planning and monitoring [21]. The capacity of current devices to now create a targeted thermal lesion within brain through the intact skull has resulted in the first FDA approval of this technology for a neurologic indication-refractory unilateral essential tremor [22].

\section{FUS: Principles of Operation}

Similar to light waves, ultrasound waves can be focused using either single element concave transducers or electronically controlled phased arrays, comprised of large numbers of much smaller piezoelectric transducers. By doing so, their energy can be concentrated up to 3 orders or magnitude in to a small ellipsoid volume $(\sim 2 \times 7 \mathrm{~mm})$ at the focus, resulting in a highintensity field. As a result, energy levels typically used in physical therapy with nonfocused transducers are capable of raising the temperature of the tissues within seconds to $60{ }^{\circ} \mathrm{C}$ or greater, when focused, to induce denaturation of cell proteins and, ultimately, coagulative necrosis. However, in the intervening tissues the width of the beam is much broader, and so corresponding intensities are lower. As a result, energy absorption is also lower and the deleterious effects of the exposures (i.e., thermal damage) do not occur [16, 23, 24].

State-of-the-art MRI-guided FUS (MRgFUS) is the current standard for image-guided FUS treatments, especially for noninvasive treatments in the brain. In early explorations on the use of FUS for clinical applications, craniectomies were necessary, owing to the presence of the skull, which generated beam distortion and energy absorption. The first hemispheric transducer arrays were developed in the 1990s to work with MRI scanners. Additionally, software was integrated into the devices for correcting phase aberrations that were generated by variable skull thickness, using preobtained computed tomography scans. These developments revolutionized the procedures, allowing for the transmission of multiple ultrasound beams across the irregular thickness of the skull to a single focus [25]. The use of MRI enables higher-resolution softtissue imaging for more accurate treatment planning. Noninvasive MR thermometry also allows for quasi-realtime temperature monitoring, important for validating that the region of treatment has received the designated thermal dose, in addition to ensuring that regions outside of the treatment zone have not been adversely affected [26].

\section{Principles of Stereotactic Surgery for Movement Disorders}

HIFU is the latest modality for stereotactic brain lesioning, which has been employed to treat movement disorders 
explored for decades. Although clearly effective, lesioning for movement disorders has been largely replaced by deep brain stimulation (DBS), which does not create an intentional brain injury. In contrast, suppression of motor abnormalities such as tremor is accomplished with DBS through continuous highfrequency stimulation [27]. Creation of bilateral brain lesions has been associated with increased risk of neurological deficits. In particular, bilateral thalamotomy for tremor related to Parkinson's disease (PD) was strongly associated with dysarthria in early studies [28]. The adjustability of DBS is an advantage over lesional surgery, as side effects of bilateral stimulation can usually be mitigated by lowering the intensity of stimulation [29].

ET and especially PD are also progressive conditions where motor symptoms worsen over time. In patients treated with DBS, the parameters of stimulation are adjusted to compensate for worsening symptoms. Disease progression may eventually result in worsening symptoms in DBS patients in spite of reprogramming [30]. Although successful open lesional repeat surgery has been performed, no patients have received repeat FUS brain lesioning at this point [31].

DBS is not without surgical complications such as intracerebral hemorrhage $(0.5-2.0 \%)$ and infection (1-3\%), as well as DBS-specific issues such as lead migration and fracture $(1-3 \%)$ and device malfunction $(1-3 \%)$ [32-34]. DBS also requires the additional surgical implantation and periodic replacement of the programmable pulse generator.

Radiosurgery has also been applied to relieve symptoms of both ET and PD, with the best results seen in lesioning for ET [35-37]. Like FUS, radiosurgery also utilizes MRIguided stereotactic methods to localize the brain target and focus an array of emitters that has been extensively used to treat brain tumors (Gamma Knife) [38]. An obstacle to widespread acceptance of this method for functional neurosurgery is the delayed effects of ionizing radiation. Although the rate of off-target effects for radiosurgery is relatively low, they can occur with a delay of days to months [39]. The accuracy and safety of radiosurgery in experienced hands is illustrated by a recent study where thalamic lesions were created bilaterally to treat patients with ET with both bilateral appendicular and axial tremor. The incidence of dysarthria, although delayed, was far lower than older lesional surgery and similar to that of DBS [40].

It is in this therapeutic environment that HIFU has been developed as a treatment of movement disorders. The goal of all of these procedures is maximal relief of motor symptoms (tremor, bradykinesia, rigidity, dystonia) without intrusion of symptoms associated with damage or stimulation of adjacent (off-target) brain regions such as dysarthria, paresthesia, weakness, ataxia, diplopia, or visual field defects.

\section{MR-Guided HIFU for Movement Disorders}

Similar to DBS, HIFU-treated patients begin their day with shaving of the head, followed by placement of a stereotactic frame using local anesthetic at the pin sites, where bruising and bleeding of the scalp can occur. They then spend approximately $1 \mathrm{~h}$ in the MRI scanner during the alignment process of the ultrasound array with the MRI. As with DBS, patients remain off medication for their movement disorder on the treatment day, to maximize motor symptoms as a target for treatment endpoint. Along with the stereotactic frame the head is covered with a silicone rubber bag filled with chilled degassed circulating water. This improves coupling of the ultrasound array to the head, and is important to reduce sonication related heating of the skull and scalp, the major technical limitation for current devices. Stereotactic surgery, including DBS placement, usually utilizes microelectrode recording to validate target location by the regional firing patterns of neuronal units. The minimally invasive strategy of MRgHIFU does not allow for physiologic recording, but both neurons and myelinated axons can be activated by nonlethal ultrasound energy with responses similar to electrical stimulation [41].

ET was the first neurologic disorder evaluated for treatment with current HIFU devices for several reasons. 1) The anatomical target [ventral intermediate nucleus (VIM) of the thalamus] is centrally located within the brain, minimizing the distortional effects of the skull on focusing ultrasound energy. 2) The VIM is a well-established target for both lesioning and DBS for reduction of tremor in medically refractory patients with either ET or PD. 3) Treatment of the VIM in ET not only results in tremor reduction, but can also substantially reduce disability in selected patients with only unilateral treatment, such as those with severe tremor in the dominant hand.

This approach has been validated by several published clinical studies that have shown significant improvement after treatment using standardized scales rating both tremor amplitude and tremor-related disability.

In an initial study, Lipsman et al. [42] treated 4 patients with medication-resistant ET, resulting in $>80 \%$ reduction on tremor scores for at least 3 months with associated functional improvement. As expected from earlier surgical studies, the only adverse neurologic effect was paresthesia on the treated hand, which persisted in 1 patient [42]. The University of Virginia group treated 15 patients with ET with similar characteristics and sonication parameters [1]. Treatment resulted in $>60 \%$ reduction in hand tremor versus baseline, with associated improvement in tremor-related disabilities in activities such as writing, drinking, and eating, which was persistent for at least 1 year. Adverse effects related to HIFU treatment included head pain, light-headedness, nausea, and a sensation of movement [43]. Thalamotomy-related adverse events included sensory changes seen in the majority of patients, but 
persisted in only 3 patients. Transient unsteadiness, weakness, and dysarthria were also observed. MRI-related abnormalities were observed within $24 \mathrm{~h}$ of sonication at the target location predicted by the real-time magnetic resonance thermography. Treated patients also showed alterations in thalamic connectivity on MRI diffusion tensor imaging sequences [44]. These observations have been validated in a recent larger multicenter, double-blind, sham-controlled pivotal study [22]. Although thalamotomy-related adverse effects with FUS have been common, none of the published studies has rated their severity in detail. In the pivotal study of the 56 treated patients, $21(38 \%)$ noted numbness or paresthesia, of which $8(14 \%)$ persisted at 12 months. Only one patient was described with a dense hypoesthesia of the dominant thumb and middle finger, categorized as a serious adverse event. Of the 20 patients with ET that our center has treated at this point (both double-blind and open-label) only one described the sensory changes as bothersome - "a sense of a burnt tongue", but there was no associated change in function, and that patient, on a global self-rating scale, was noted as much improved after treatment. Similar comments apply to thalamotomy-related weakness in the pivotal study where a single patient with persistent weakness was not considered to have a serious adverse event. In the earlier study by the University of Virginia group discussed above, 1 patient had subjective hand weakness but showed unchanged grip strength with dynamometry.

MRgHIFU has also been targeted to relevant white matter tracts that have rarely been approached by open movement disorder surgical methods. The cerebellothalamic tract was the target in a group of 21 consecutive patients with severe refractory ET, with comparable improvement in tremor severity and disability to studies targeting the VIM. Adverse events of treatment were relatively mild and nonserious. Notably, this study included the first 3 patients to receive bilateral (staged) MRgHIFU brain lesions for a movement disorder [45]. A desire to reduce complications associated with thalamic damage was the rationale for targeting the cerebellothalamic tract with HIFU. Bilateral surgical lesioning for movement disorders has rarely been performed after early experiences in the 1960s, where unexpected severe dysarthria or imbalance was frequently observed. The observation that the patients treated with HIFU had a relatively low level of worsening of preexisting gait instability (4/21 transient, $1 / 21$ permanent) and were also without dysarthria is consistent with results of the study of bilateral thalamotomy using radiosurgery for ET [40].

These MRgHIFU studies have utilized a device with > 1000 sonic energy-emitting elements (Exblate, Insightec; Fig. 1). Attempts to treat patients with the current device ( $650 \mathrm{kHz}$ frequency of sonic energy), designed for a high level of accuracy of target sonication, sometimes fail to attain sufficient thermal doses at the target for lesioning. This is usually due to skull characteristics that can raise sonic energy attenuation, which ultimately limits energy deposition at the target
$[46,47]$. In the pivotal trial for ET, patients underwent a screening noncontrast head computed tomography, from which the skull density index (the ratio of density of cortical to cancellous bone) was calculated. Only patients with a skull density index of $>0.45$ were treated.

As with previous surgical studies, VIM has also been targeted with HIFU for relief of tremor associated with PD. An initial study of 7 patients with severe refractory tremor associated with PD demonstrated immediate abolition of contralateral arm tremor that persisted for at least 3 months, with mild neurologic deficits that did not persist [48]. MRgHIFU thalamotomy gave reduction of PD tremor in a blinded sham controlled study, recently reported in abstract form. However, tremor reduction was not statistically significant in this small group of patients [49]. There has been a small experience with tremor from other conditions. Two patients have been reported with tremor associated with the fragile $\mathrm{X}$ ataxia syndrome with substantial reduction of tremor after FUS-mediated thalamotomy $[50,51]$.

Targets other than the thalamus have been treated with HIFU for relief of other signs and symptoms of PD. Unilateral lesions were created with HIFU in 13 patients with PD targeting the fiber tracts exiting the pallidum on route to thalamus (pallidothalamic tract), including the fasiculus lenticularis and ansa lenticularis [2]. Although the initial patients treated had rapid return of PD symptoms associated with insufficient increases in target temperature, increasing the ultrasound energy resulted in a $60 \%$ reduction in Unified Parkinson's disease rating scale scores in 9 subsequent patients. This group of patients was heterogeneous with regard to aspects of PD (tremor, bradykinesia, dyskinesias) that dominated their clinical picture. Improvement persisted during the 3-month follow-up. As in the vast majority of MRgHIFU studies, postprocedure MRI showed a lesion at the site that received adequate thermal energy (Fig. 2, from Magara et al. [2]). These MRI changes diminish after time, but reversal of lesions visible with MRI does not correlate well with the duration of clinical effect.

The globus pallidus interna (GPi) is another wellestablished target for both surgical lesioning and DBS of PD $[52,53]$. Lesional surgery to the GPi not only has been shown to improve cardinal signs of PD such as tremor, bradykinesia and rigidity, but is also particularly effective in reducing the abnormal movements that develop after years of treatment with L-dopa [L-dopa-induced dyskinesias (LIDs)] [54]. Complications of lesions of the GPi have included hemiparesis and visual field deficits due the proximity of the internal capsule and optic tract [55]. Patients with PD have also begun to be treated with HIFU targeted to the GPi. The first reported case, a patient with PD and intrusive dyskinesias, was successfully treated with an MRgHIFU unilateral pallidotomy. This patient experienced a $76 \%$ reduction in the severity of motor signs in the "off" medication state, as well as a 

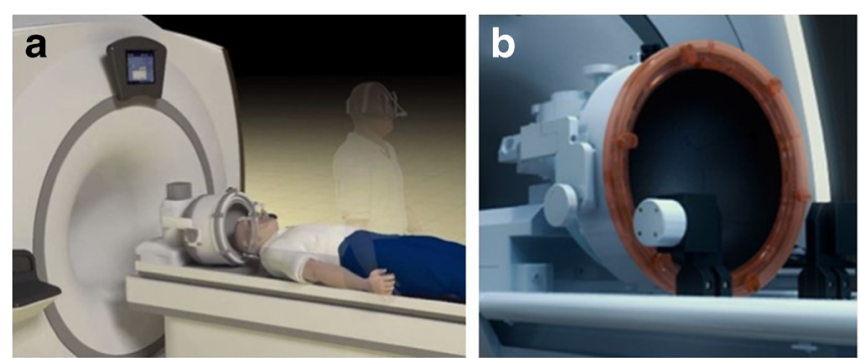

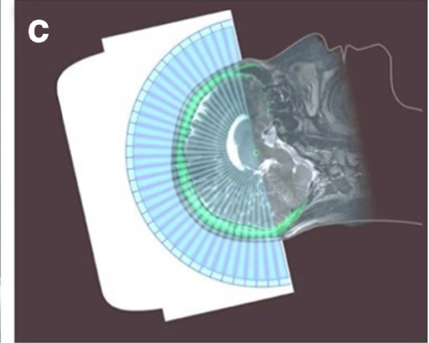

bladder and the face of the transducer array to maximize coupling and reduce heating effects. (C) A schematic 2-dimensional representation of the multiple ultrasound beams focused, noninvasively, through the skull (bright green) to a single target. The image of the skull is obtained from a prior computed tomography scan that is mechanically registered to the magnetic resonance image. Information from the skull is utilized by the planning software to correct for aberrations to the beam paths, and accurately position the focus at the desired target. Images obtained and adapted with permission from Insightec Ltd, Israel
53\% reduction in dyskinesia ratings even 6 months after the procedure. As with surgical pallidotomy some improvement was even seen ipsilateral to the treated hemisphere, without any off-target neurologic adverse effects [56]. Our center is currently part of a safety and feasibility study of MRgHIFU pallidotomy with plans for a multicenter phase II study. Treated patients have highly asymmetric motor signs and are L-dopa responsive but have significant disability from LIDs.

Although the subthalamic nucleus (STN) is the most common target for DBS treatment of PD, it was rarely targeted for stereotactic surgery in the past. The reluctance is based on lesion studies in primates and experience in patients after stroke, where destruction of the STN results in dramatic involuntary movements such as hemiballismus [57]. A large study in which stereotactic surgical lesions were created in the STN resulted in improvement in PD motor symptoms but a significant incidence of hemiballismus [58]. Whether a more controlled approach using MRgHIFU could allow for safe and effective lesioning of the STN remains to be determined.

The majority of patients with PD who are currently treated with DBS undergo bilateral procedures. Although unilateral pallidotomy results in both unilateral and contralateral reduction of LIDS, increased benefit has been reported with bilateral pallidal lesions in bilateral motor symptoms, including gait $[59,60]$. Bilateral GPi lesions have also been associated with worsening of dysarthria and drooling in patients with PD, although the degree of increased risk over unilateral lesions appears small in more contemporary studies [61, 62]. As with bilateral thalamotomy, further evaluation of both safety and

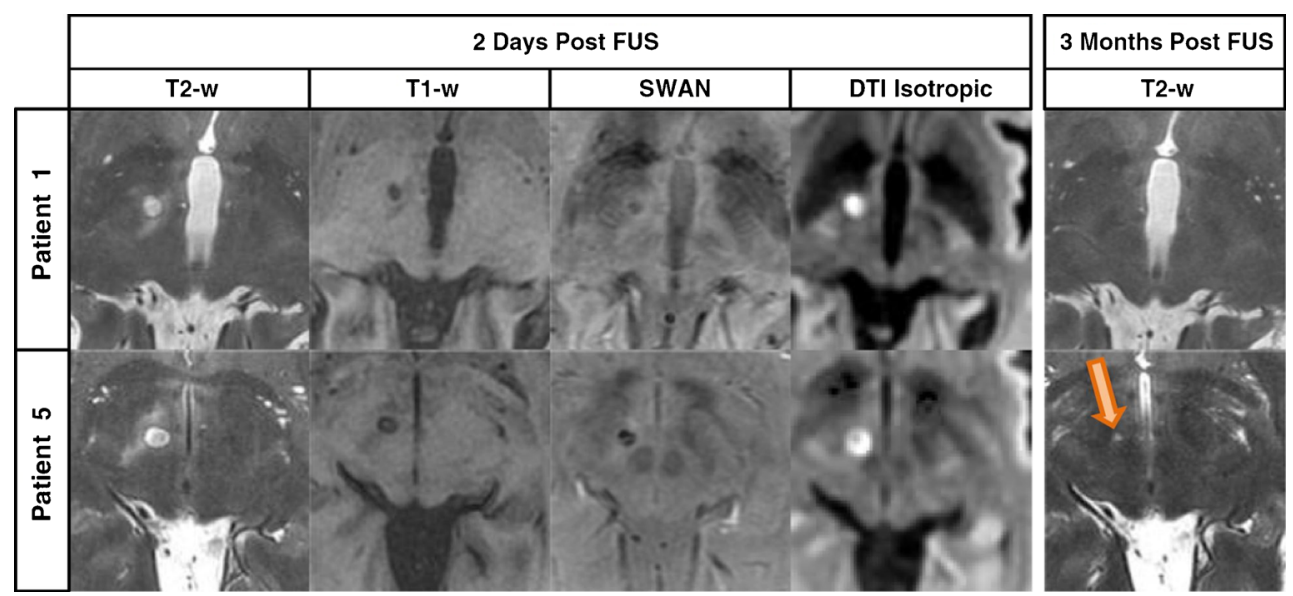

Fig. 2 Magnetic resonance imaging (MRI) of focused ultrasoundinduced lesions. A comparison of the different MRI scans available to visualize thermally induced lesions in the pallidothalamic tract in 2 different patients undergoing MRI-guided focused ultrasound-mediated pallidothalamic tractotomy, and at 2 different times post-treatment. At
3 months post-treatment, a lesion can still be seen only in patient 5 (arrow). $\mathrm{T} 2-\mathrm{w}=\mathrm{T} 2$-weighted; $\mathrm{T} 1-\mathrm{w}=\mathrm{T} 1$-weighted; SWAN $=\mathrm{T} 2$ star weighted angiography; DTI = diffusion tensor imaging. (Reprinted with permission from Magara et al [2]) 
efficacy of MRgHIFU bilateral pallidotomy will be needed before the potential of this strategy for the majority of PD surgical candidates is known.

Although MRgHIFU can cause lesion-related neurologic deficits, unlike open stereotactic surgical approaches to modify brain function (including DBS), intracerebral hemorrhage or infection have not been reported in any of the studies discussed above. At this point $>400$ patients have been treated with MRgHIFU-based functional brain ablation without these serious complications (Insightec, personal communication).

Movement disorders have not been the only brain conditions attempted to be treated with HIFU. From a theoretical viewpoint, any condition amenable to stereotactic brain lesioning or DBS could be approached with MRgHIFU. Chronic neuropathic pain has been successfully treated in the past with open stereotactic radiofrequency ablation of the central thalamus [63]. Similar improvement has been reported with MRgHIFU central lateral thalamotomy (12 patients), including in patients with bilateral procedures [3]. Refractory obsessive compulsive disorder has been a target condition for both stereotactic lesioning and DBS [64]. A pilot study has shown clinical improvement with bilateral MRgHIFUcreated lesions in the anterior limb of the internal capsule in this patient group [65]. A preclinical study has demonstrated the feasibility of performing FUS-mediated ventriculostomy, with implications for the development of a less invasive method for the treatment of obstructive hydrocephalus [66].

\section{FUS-Enhanced Delivery: Opening the BBB}

Whereas FUS exposures for ablation are carried out in continuous mode for tissue destruction, FUS exposures in pulsed mode (pFUS) are nondestructive, owing to their lower temporal averaged intensities [67, 68]. pFUS exposures also allow for cooling to occur between pulses, further reducing temperature increases [69]. Employing pFUS exposures using 5\% duty cycles, for example, where 50-ms pulses are provided once a second, have been shown to generate temperature elevations of only $4{ }^{\circ} \mathrm{C}$ to $5{ }^{\circ} \mathrm{C}[70,71]$. Instead of heat generation, these exposures are capable of creating a number of mechanical effects, most notably for nondestructively increasing vascular permeability to improve the delivery of therapeutic agents. This has been demonstrated in a variety of solid tumor models [72-74], as well as in acute and chronic clot models $[75,76]$. However, the majority of pFUS studies have involved increasing the permeability of the BBB to enhance or enable the delivery of agents to the brain.

The specialized endothelia of the brain have continuous tight junctions that form the BBB, limiting the movement of many types of therapeutics from the bloodstream into brain. Strategies that have been developed to open or bypass the BBB include hyperosmotic solutions of mannitol and carrier molecules that are transported across brain endothelia [77-79]. Studies by Hynynen, McDannold, and colleagues [80-83] initially demonstrated that FUS applied during the circulation of microbubble suspensions (FDA-approved ultrasound contrast agents) can create an MRI-targeted region of transient and safe disruption of the BBB. This allows large therapeutics to enter the brain from the systemic circulation, including: antibodies, growth factors, nanoparticles, nucleic acids, viral vectors, and cells [84-93]. The lower-intensity pulsed FUS exposures activate the microbubbles into a state of stable oscillations (i.e., noninertial cavitation), causing transient separation of endothelial tight junctions - the basis for the BBB $[82,93]$. The procedure can create transient (hours) opening of the BBB, sufficient to allow extravasation of large therapeutics without pathology or entry of blood components [94-96].

The first direct application of this strategy to neurologic disease is in brain tumor therapy. In preclinical models of brain metastatic breast cancer, FUS-mediated BBB opening substantially improved the efficacy of the antihuman epidermal growth factor 2 monoclonal antibody trastuzumab [97]. This therapy, although effective for human epidermal growth factor 2-positive breast cancer, is ineffective for brain metastasis because its molecule size prohibits its passage through the BBB. Clinical trials of FUS opening to enhance chemotherapy (doxorubicin) of brain tumors are currently enrolling, with the first patient safely undergoing FUS-mediated BBB opening in November 2015. The method can even allow very large therapeutics, such as antitumor immune cells, to enter a brain tumor $[6,8]$. Although the BBB is relatively disrupted in many brain tumors, MRgFUS can target tumor extensions into surrounding, invasive rim that do not enhance with MRI contrast agents such as gadolinium, with the goal of improving chemotherapy to these refractory areas [98]. This approach to FUS therapy of brain tumors may have less potential risk than a direct thermal tumor ablation. The initial experience with HIFU ablation of malignant glioma resulted in hemorrhagic complications [99].

Preclinical studies have used MRgFUS to improve the delivery of growth factors and their genes in the treatment of PD. The delivery of glial cell-derived neurotrophic factor (GDNF) and the related factor neurturin from the blood was improved in rodents with the use of this procedure [100, 101]. Gene therapy with GDNF has been successful in restoring dopamine metabolism and reversing motor abnormalities in a toxin-induced rat model of PD [102]. In this study, a plasmid expressing GDNF was preloaded into the microbubbles to enhance its concentration in the region of FUS-mediated BBB opening. Viral vectors carrying potentially beneficial genes can also be delivered to brain from an intravenous injection after FUS-mediated opening of the BBB [87, 103]. After more than a decade of experience in animals that includes nonhuman primates, this method is accumulating 
substantial data supporting its safety, including the use of repeated treatments, an approach essential to the continued treatment of chronic progressive neurologic disease [104, 105]. Development of gene therapy for PD has been hampered by the difficulty translating promising preclinical results in animal models to successful clinical trials [106-109]. Improving the delivery and distribution of the gene vector with FUS may be one useful approach to help bring this complex multifactorial form of brain therapy to clinical practice.

Treatment of Alzheimer's disease (AD) is also a potential goal with FUS-mediated opening of the BBB. Studies in mouse models of $\mathrm{AD}$ have demonstrated both reduction in brain amyloid burden and behavioral improvement using this strategy, coupled with either injected or endogenous antiamyloid antibodies [110-112]. These positive results occurred without evidence of brain hemorrhage, a clear concern when considering the coexistence and amyloid angiopathy in AD. The development of clinical amyloid-based nuclear medicine scans make a planned pilot study in humans feasible, with the aim of determining if FUS can reduce the amyloid burden in a local brain region. One of the animal studies demonstrated how moving the target of sonication through the brain (scanning FUS) could be a potential useful strategy for treatment of a large brain volume [113].

Using FUS to open the BBB has even been applied to therapeutics as large as cells in experimental animals. Stem cells have been found in brain regions, where FUS-mediated BBB opening was followed by intracarotid injection, while lymphocytes will enter treated brain regions even after intravenous injections [6, 7]. Stem cell therapy could also be advanced through another novel use of FUS, reported to stimulate endogenous brain stem cell proliferation [114].

Delivery of large therapeutics across the BBB has always been limited by the inefficiency of the transfer where accumulation of $1 \%$ to $2 \%$ in the brain of the total injected into the blood is a true accomplishment [79]. Within safe parameters, BBB opening may last only a few hours, and the amount of the therapeutic entering brain is usually much less. Studies of molecular or cellular therapies usually find that $<0.1 \%$ of the injected agent can be detected in the sonicated region of brain after MRgFUS-mediated opening of the BBB [7, 97]. Our group has attempted to address this issue by combining an FUS-based method with a complimentary strategy known as magnetic targeting or attraction. This method is based on attracting super paramagnetic iron oxide containing nanoparticles (SPION) to an applied magnetic field [115]. Molecular therapeutics such as beneficial genes can be coupled to the particles or, in the case of our own work, stem cells can be loaded with SPIONs that they engulf in culture [116, 117]. Our preliminary work indicates that stem cells loaded with SPIONs have a much greater likelihood of entering the brain from the blood after FUS-mediated opening of the BBB is combined with the application of a powerful external magnet [118].
Delivery of molecular and cellular therapeutics through opening of the BBB has the potential to be both safer and more effective than the current method in both humans and experimental animals of intracerebral needle injection. A major limitation of this approach in the case of cell-based therapy for $\mathrm{PD}$ is the poor migration of stem cells from the injection site into the large and unfavorable environment of the adult human brain [119-122]. Although inefficient, opening the BBB allows the cells to be widely distributed throughout the target region using the brains natural route of delivery - the microvasculature.

FUS is also being investigated to further improve convectionenhanced delivery using intracerebral injection, which is the current standard for delivery of protein and gene therapy to the brain. Applying energy to brain tissue with FUS improves the spread of injectates, including nanoparticles, after convection-enhanced delivery [123, 124].We recently demonstrated that pulsed ultrasound exposures can safely enlarge both the extracellular and perivascular spaces in ex vivo brain tissue. Generating these effects was subsequently shown to significantly enhance the diffusion of densely PEGylated nanoparticles as large as $500 \mathrm{~nm}$ when injected directly into the cortex following the exposures [125]. Similar mechanisms may be involved in the enhancement of transnasal delivery of proteins after sonication of brain [126].

The combination of circulating microbubbles and FUS has also been recently explored as an alternative method of brain lesioning to HIFU-mediated thermal ablation. Unlike FUSmediated opening of the BBB, the goal is to use higher levels of sonic energy to destroy the microvasculature creating a cystic lesion [127]. This method may be particularly applicable to the ablation of epileptic foci, where the proximity of target cortical regions to the skull makes thermal ablation with HIFU problematic [128].

\section{FUS-Mediated Neuromodulation}

Excitation or inhibition of neural activity, termed neuromodulation, has been studied with pFUS exposures at intensities that are substantially (i.e., orders of magnitude) lower than even those used for drug delivery applications. In a mouse model of somatomotor response, for example, transcranial pFUS exposures were shown to elicit responses in the forelimb, measured by electromyography. By varying the intensity and the pulse width of the exposures, the effects of these changes were characterized for the duration and strength of the contractions, as well as their latency [129]. A follow-up study by the same group then showed that exposures in the rostral and caudal regions of the motor cortex could selectively stimulate motor activity in the neck and tail regions, respectively [9]. 
In addition to activation, neuromodulation studies have also demonstrated how pFUS exposures can suppress neuronal activity. In a chemically induced epilepsy model in rats, pFUS exposures in the thalamus were shown to significantly suppress acute epileptic electroencephalogram activity [12]. Another study in rabbits found that pFUS exposures can also significantly suppress visual activity induced by light stimulation. This was measured in visually evoked potentials by the p30 component, where suppression was observed for almost $10 \mathrm{~min}$ postexposure [11]. In one study the neuromodulatory effects of low-intensity pFUS provided evidence for the potential for treating disorders of consciousness. Exposures, carried out in the thalamus of rats, anesthetized with an intraperitoneal injection of ketamine and xylazine, reduced the time for emergence of voluntary movement, as well as the time to demonstrate a pinch response [130].

The studies described here are representative of a host of others showing anatomical specificity of pFUS-induced neuromodulation. In one study, for example, pFUS exposures were carried out in the motor cortex of rats, immediately following the administration of 2-deoxy-[18F]fluoro-D-glucose (FDG). Normally employed to image enhanced glucose metabolism by positron emission tomography, increased FDG signals were observed in the targeted region, attesting to the location of stimulation. In another study, the accuracy of targeting MRgFUS exposures in the motor cortex of rabbit brains was similarly verified using functional MRI [41]. The mechanism by which ultrasound can induce neurostimulation has yet to be determined. Preliminary investigations to date have pointed to triggering of voltage-dependent somatic and presynaptic $\mathrm{Ca}^{+}$transients in neurons, where stimulated regions correspond to higher densities of ${\mathrm{c}-\mathrm{fos}^{+}}^{+}$cells [131]. However, there is a general consensus that pFUS-induced neuromodulation occurs through nonthermal mechanisms of ultrasound due to the marginal and biological inconsequential temperature elevations associated with pFUS [11, 129]. To date, the most plausible mechanism proposed has been intramembrane cavitation within the bilayer membrane [132], where selective cell-type mechanisms may exist that, for example, can boost charge accumulation in affected T-type calcium channels in low threshold spiking interneurons [133]. Depending on the application, pFUS effects on neuromodulation can endure for up to hours or even days, in the absence of producing any damaging effects to the exposed tissue [11, 12].

Regarding the use of pFUS for neuromodulatory applications, comparably much simpler devices have been proposed and evaluated. Indeed, a number of studies have demonstrated how single-element FUS transducers, positioned directly on the scalp, can been used for effective transcranial neuromodulation in humans [134, 135]. However, such a device would require some type of guidance, presumably similar to the manner for transcranial magnetic stimulation.
Over the last decade the number of publications of animal and human studies utilizing some form of therapeutic ultrasound has expanded exponentially. This progress is a reflection of a growing understanding of this new technology among a community of investigators that include medical physicists, biomedical engineers, neuroradiologists, neurophysiologists, neurosurgeons, psychiatrists, and neurologists. The need for translating new technologies and concepts into clinical therapies, and the growing appreciation of a "teamscience" approach, will bring the application of therapeutic ultrasound to neurological disease into focus.

Required Author Forms Disclosure forms provided by the authors are available with the online version of this article.

\section{References}

1. Elias WJ, Huss D, Voss T, et al. A pilot study of focused ultrasound thalamotomy for essential tremor. N Engl J Med 2013;369: 640-648.

2. Magara A, Bühler R, Moser D, Kowalski M, Pourtehrani P, Jeanmonod D. First experience with MR-guided focused ultrasound in the treatment of parkinson's disease. J Ther Ultrasound 2014;2:11.

3. Jeanmonod D, Werner B, Morel A, et al. Transcranial magnetic resonance imaging-guided focused ultrasound: Noninvasive central lateral thalamotomy for chronic neuropathic pain. Neurosurg Focus 2012;32:E1.

4. Wei KC, Chu PC, Wang HY, Huang CY, Chen PY, Tsai HC, Lu YJ, Lee PY, Tseng IC, Feng LY, Hsu PW, Yen TC, Liu HL (2013) Focused ultrasound-induced blood-brain barrier opening to enhance temozolomide delivery for glioblastoma treatment: a preclinical study, PLoS One 8, e58995.

5. Treat L, McDannold N, Zhang Y, et al. Improved anti-tumor effect of liposomal doxorubicin after targeted blood-brain barrier disruption by MRI-guided focused ultrasound in rat glioma. Ultrasound Med Biol 2012;38:1716-1725.

6. Alkins R, Burgess A, Ganguly M, et al. Focused ultrasound delivers targeted immune cells to metastatic brain tumors. Cancer Res 2013;73:1892-1899.

7. Burgess A, Ayala-Grosso CA, Ganguly M, Jordão JF, Aubert I, Hynynen K. Targeted delivery of neural stem cells to the brain using MRI-guided focused ultrasound to disrupt the blood-brain barrier. PLOS ONE 2011;6:e27877.

8. Alkins R, Burgess A, Kerbel R, Wels WS, Hynynen K. Early treatment of HER2-amplified brain tumors with targeted NK-92 cells and focused ultrasound improves survival. Neuro Oncol 2016;18:974-981.

9. Kim H, Lee SD, Chiu A, Yoo SS, Park S. Estimation of the spatial profile of neuromodulation and the temporal latency in motor responses induced by focused ultrasound brain stimulation. Neuroreport 2014;25:475-479.

10. King RL, Brown JR, Newsome WT, Pauly KB. Effective parameters for ultrasound-induced in vivo neurostimulation. Ultrasound Med Biol. 2013 Feb;39(2):312-31.

11. Yoo S, Bystritsky A, Lee J, et al. Focused ultrasound modulates region-specific brain activity. Neuroimage 2011;56:1267-1275.

12. Min B, Bystritsky A, Jung K, et al. Focused ultrasound-mediated suppression of chemically-induced acute epileptic EEG activity. BMC Neurosci 2011;12:23. 
13. Lynn JG, Zwemer RL, Chick AJ, Miller AE. A new method for the generation and use of focused ultrasound in experimental biology. J Gen Physiol. 1942;26:179-193.

14. Frenkel V. Image-guided focused ultrasound: endless possibilities for non-invasive therapy in the 21st century. JSM Biotechnol Biomed Eng 2013;1:1001.

15. Warden SJ, Fuchs RK, Kessler CK, Avin KG, Cardinal RE, Stewart RL. Ultrasound produced by a conventional therapeutic ultrasound unit accelerates fracture repair. Phys Ther. 2006;86: 1118-1127.

16. Kennedy JE. High-intensity focused ultrasound in the treatment of solid tumours. Nat Rev Cancer 2005;5:321-327.

17. Kong CY, Meng L, Omer ZB, et al. MRI-guided focused ultrasound surgery for uterine fibroid treatment: a cost-effectiveness analysis. AJR Am J Roentgenol. 2014;203:361-371.

18. Roubidoux MA, Yang W, Stafford RJ. Image-guided ablation in breast cancer treatment. Tech Vasc Interv Radiol 2014;17:49-54.

19. Napoli A, Anzidei M, Marincola BC, et al. MR imaging-guided focused ultrasound for treatment of bone metastasis. Radiographics 2013;33:1555-1568.

20. Limani K, Aoun F, Holz S, Paesmans M, Peltier A, van Velthoven R. Single high intensity focused ultrasound session as a whole gland primary treatment for clinically localized prostate cancer: 10-year outcomes. Prostate Cancer.2014;2014:186782.

21. Jolesz FA, McDannold NJ. Magnetic resonance-guided focused ultrasound: a new technology for clinical neurosciences. Neurol Clin 2014:32:253-269.

22. Elias WJ, Lipsman N, Ondo WG, et al. A randomized trial of focused ultrasound thalamotomy for essential tremor. $N$ Engl $J$ Med 2016;375:730-739.

23. O'Brien WD. Ultrasound-biophysics mechanisms. Prog Biophys Mol Biol 2007;93:212-255.

24. Huisman M, van den Bosch MA. MR-guided high-intensity focused ultrasound for noninvasive cancer treatment. Cancer Imaging 2011;11:S161-S166.

25. Clement GT, White PJ, King RL, McDannold N, Hynynen K. A magnetic resonance imaging-compatible, large-scale array for trans-skull ultrasound surgery and therapy. J Ultrasound Med 2005;24:1117-1125.

26. Hynynen K, Jolesz FA. Demonstration of potential noninvasive ultrasound brain therapy through an intact skull. Ultrasound Med Biol 1998;24:275-283.

27. Wichmann T, DeLong MR. Deep brain stimulation for movement disorders of basal ganglia origin: restoring function or functionality? Neurotherapeutics 2016;13:264-283.

28. Selby G. Stereotactic surgery for the relief of parkinson's disease: Part 2. an analysis of the results in a series of 303 patients (413 operations). J Neurol Sci 1967;5:343-375.

29. Tasker RR. Deep brain stimulation is preferable to thalamotomy for tremor suppression. Surg Neurol 1998;49:145-153.

30. Castrioto A, Lozano AM, Poon Y, Lang AE, Fallis M, Moro E. Ten-year outcome of subthalamic stimulation in parkinson disease: a blinded evaluation. Arch Neurol 2011;68:1550-1556.

31. Nagaseki Y, Shibazaki T, Hirai T, et al. Long-term follow-up results of selective VIM-thalamotomy. J Neurosurg 1986;65:296302.

32. Patel DM, Walker HC, Brooks R, Omar N, Ditty B, Guthrie BL. Adverse events associated with deep brain stimulation for movement disorders: analysis of 510 consecutive cases. Neurosurgery 2015;11(Suppl. 2):190-199.

33. Sillay KA, Larson PS, Starr PA. Deep brain stimulator hardwarerelated infections: Incidence and management in a large series. Neurosurgery 2008;62:360-366.

34. Fenoy AJ, Simpson Jr RK. Risks of common complications in deep brain stimulation surgery: management and avoidance: clinical article. J Neurosurg 2014;120:132-139.
35. Campbell AM, Glover J, Chiang VL, Gerrard J, James BY. Gamma knife stereotactic radiosurgical thalamotomy for intractable tremor: a systematic review of the literature. Radiother Oncol 2015;114:296-301.

36. Young RF, Li F, Vermeulen S, Meier R. Gamma knife thalamotomy for treatment of essential tremor: long-term results: clinical article. J Neurosurg 2010;112:1311-1317.

37. Hua Z, Guodong G, Qinchuan L, Yaqun Z, Qinfen W, Xuelian W. Analysis of complications of radiofrequency pallidotomy. Neurosurgery 2003;52:89-101.

38. Specht HM, Combs SE. Stereotactic radiosurgery of brain metastases. J Neurosurg Sci 2016;60:357-366.

39. Yamamoto M, Kawabe T, Higuchi Y, et al. Delayed complications in patients surviving at least 3 years after stereotactic radiosurgery for brain metastases. Int J Radiat Oncol Biol Phys 2013;85:53-60.

40. Young RF, Hesselgesser RD, Ahn E, Vermeulen S, Li F, Lee J. Bilateral gamma knife thalamotomy for treatment of axial tremor. Transl Cancer Res 2014;3:525-529.

41. Tyler WJ, Tufail Y, Finsterwald M, Tauchmann ML, Olson EJ, Majestic C. Remote excitation of neuronal circuits using low-intensity, low-frequency ultrasound. PLOS ONE 2008;3:e3511.

42. Lipsman N, Schwartz ML, Huang Y, et al. MR-guided focused ultrasound thalamotomy for essential tremor: a proof-of-concept study. Lancet Neurol 2013;12:462-468.

43. Huss DS, Dallapiazza RF, Shah BB, Harrison MB, Diamond J, Elias WJ. Functional assessment and quality of life in essential tremor with bilateral or unilateral DBS and focused ultrasound thalamotomy. Mov Disord 2015;30:1937-1943.

44. Wintermark M, Huss DS, Shah BB, et al. Thalamic connectivity in patients with essential tremor treated with MR imaging-guided focused ultrasound: in vivo fiber tracking by using diffusiontensor MR imaging. Radiology 2014;272:202-209.

45. Gallay MN, Moser D, Rossi F, et al. Incisionless transcranial MRguided focused ultrasound in essential tremor: cerebellothalamic tractotomy. $J$ Ther Ultrasound.2016;4:1.

46. Chang WS, Jung HH, Zadicario E, et al. Factors associated with successful magnetic resonance-guided focused ultrasound treatment: efficiency of acoustic energy delivery through the skull. $J$ Neurosurg 2016;124:411-416.

47. Jung H, Kim S, Roh D, et al. Bilateral thermal capsulotomy with MR-guided focused ultrasound for patients with treatmentrefractory obsessive-compulsive disorder: a proof-of-concept study. Mol Psychiatry 2015;20:1205-1211.

48. Schlesinger I, Eran A, Sinai A, et al. MRI guided focused ultrasound thalamotomy for moderate-to-severe tremor in parkinson's disease. Park Dis 2015;2015:219149.

49. Bond AE, Dallapiazza R, Huss D, et al. 132 A randomized, shamcontrolled trial of transcranial magnetic resonance-guided focused ultrasound thalamotomy trial for the treatment of tremor-dominant, idiopathic parkinson disease. Neurosurgery 2016;63:154.

50. Cerquera C, Rumià J, Herrera JM, Moreno V, Bargalló N, Valldeoriola F. A single case report of MR-guided focused ultrasound thalamotomy for tremor in fragile $\mathrm{x}$-associated tremor/ ataxia. Parkinsonism Relat Disord 2016;28:159-160.

51. Fasano A, Sammartino F, Llinas M, Lozano AM. MRI-guided focused ultrasound thalamotomy in fragile X-associated tremor/ ataxia syndrome. Neurology 2016;87:736-738.

52. Lang AE, Lozano AM, Montgomery E, Duff J, Tasker R, Hutchinson W. Posteroventral medial pallidotomy in advanced parkinson's disease. N Engl J Med 1997;337:1036-1043.

53. Vitek JL. Deep brain stimulation for parkinson's disease. A critical re-evaluation of STN versus GPi DBS. Stereotact Funct Neurosurg 2002;78:119-131.

54. Munhoz RP, Cerasa A, Okun MS. Surgical treatment of dyskinesia in Parkinson's disease. Front Neurol 2014;5:65. 
55. Iacono RP, Shima F, Lonser RR, Kuniyoshi S, Maeda G, Yamada $\mathrm{S}$. The results, indications, and physiology of posteroventral pallidotomy for patients with parkinson's disease. Neurosurgery 1995;36:1118-1127.

56. Na YC, Chang WS, Jung HH, Kweon EJ, Chang JW. Unilateral magnetic resonance-guided focused ultrasound pallidotomy for parkinson disease. Neurology 2015;85:549-551.

57. Lee M, Marsden C. Movement disorders following lesions of the thalamus or subthalamic region. Mov Disord 1994;9:493-507.

58. Alvarez L, Macias R, Pavon N, et al. Therapeutic efficacy of unilateral subthalamotomy in parkinson's disease: results in 89 patients followed for up to 36 months. J Neurol Neurosurg Psychiatry 2009;80:979-985.

59. Parkin SG, Gregory RP, Scott R, et al. Unilateral and bilateral pallidotomy for idiopathic parkinson's disease: a case series of 115 patients. Mov Disord 2002;17:682-692.

60. Counihan TJ, Shinobu LA, Eskandar EN, Cosgrove GR, Penney $\mathrm{JB}$, Jr. Outcomes following staged bilateral pallidotomy in advanced parkinson's disease. Neurology 2001;56:799-802.

61. Siegel KL, Metman LV. Effects of bilateral posteroventral pallidotomy on gait of subjects with parkinson disease. Arch Neurol 2000;57:198-204.

62. Intemann PM, Masterman D, Subramanian I, et al. Staged bilateral pallidotomy for treatment of parkinson disease. J Neurosurg 2001;94:437-444.

63. Weigel R, Krauss JK. Center median-parafascicular complex and pain control. review from a neurosurgical perspective. Stereotact Funct Neurosurg 2004;82:115-126.

64. Brown LT, Mikell CB, Youngerman BE, Zhang Y, McKhann GM, Sheth SA. Dorsal anterior cingulotomy and anterior capsulotomy for severe, refractory obsessive-compulsive disorder: a systematic review of observational studies. J Neurosurg 2016;124:77-89.

65. Jung HH, Chang WS, Rachmilevitch I, Tlusty T, Zadicario E, Chang JW. Different magnetic resonance imaging patterns after transcranial magnetic resonance-guided focused ultrasound of the ventral intermediate nucleus of the thalamus and anterior limb of the internal capsule in patients with essential tremor or obsessivecompulsive disorder. J Neurosurg 2015;122:162-168.

66. Alkins R, Huang Y, Pajek D, Hynynen K. Cavitation-based third ventriculostomy using MRI-guided focused ultrasound. $J$ Neurosurg 2013;119:1520-1529.

67. Hancock HA, Smith LH, Cuesta J, et al. Investigations into pulsed high-intensity focused ultrasound-enhanced delivery: preliminary evidence for a novel mechanism. Ultrasound Med Biol 2009;35: 1722-1736.

68. O'Neill BE, Vo H, Angstadt M, Li KP, Quinn T, Frenkel V. Pulsed high intensity focused ultrasound mediated nanoparticle delivery: mechanisms and efficacy in murine muscle. Ultrasound Med Biol 2009;35:416-424.

69. Frenkel V. Ultrasound mediated delivery of drugs and genes to solid tumors. Adv Drug Deliv Rev 2008;60:1193-1208.

70. Frenkel V, Etherington A, Greene M, et al. Delivery of liposomal doxorubicin (doxil) in a breast cancer tumor model: investigation of potential enhancement by pulsed-high intensity focused ultrasound exposure. Acad Radiol 2006;13:469-479.

71. Patel PR, Luk A, Durrani A, et al. In vitro and in vivo evaluations of increased effective beam width for heat deposition using a split focus high intensity ultrasound (HIFU) transducer. Int $J$ Hyperthermia 2008;24:537-549.

72. Dittmar KM, Xie J, Hunter F, et al. Pulsed high-intensity focused ultrasound enhances systemic administration of naked DNA in squamous cell carcinoma model: initial experience 1. Radiology 2005;235:541-546.

73. Ziadloo A, Xie J, Frenkel V. Pulsed focused ultrasound exposures enhance locally administered gene therapy in a murine solid tumor model. J Acoust Soc Am 2013;133:1827-1834.
74. Poff JA, Allen CT, Traughber B, et al. Pulsed high-intensity focused ultrasound enhances apoptosis and growth inhibition of squamous cell carcinoma xenografts with proteasome inhibitor bortezomib 1. Radiology 2008;248:485-491.

75. Stone MJ, Frenkel V, Dromi S, et al. Pulsed-high intensity focused ultrasound enhanced tPA mediated thrombolysis in a novel in vivo clot model, a pilot study. Thromb Res 2007;121:193-202.

76. Abi-Jaoudeh N, Pritchard WF, Amalou H, et al. Pulsed highintensity-focused US and tissue plasminogen activator (TPA) versus TPA alone for thrombolysis of occluded bypass graft in swine. $J$ Vasc Interv Radiol 2012;23:953-961.

77. Gonzales-Portillo GS, Sanberg PR, Franzblau M, et al. Mannitolenhanced delivery of stem cells and their growth factors across the Blood-Brain barrier. Cell Transplant 2014;23:531-539.

78. Doolittle ND, Muldoon LL, Culp AY, Neuwelt EA. Delivery of chemotherapeutics across the blood-brain barrier: challenges and advances. Adv Pharmacol 2014;71:203-243.

79. Pardridge WM, Boado RJ. Reengineering biopharmaceuticals for targeted delivery across the blood-brain barrier. Methods Enzymol 2012;503:269-292.

80. Hynynen K. Focused ultrasound for blood-brain disruption and delivery of therapeutic molecules into the brain. Expert Opin Drug Deliv 2007;4:27-35.

81. Hynynen K. Macromolecular delivery across the blood-brain barrier. Methods Mol Biol 2009;480:175-185.

82. Sheikov N, McDannold N, Vykhodtseva N, Jolesz F, Hynynen K. Cellular mechanisms of the blood-brain barrier opening induced by ultrasound in presence of microbubbles. Ultrasound Med Biol 2004;30:979-989.

83. McDannold N, Vykhodtseva N, Hynynen K. Use of ultrasound pulses combined with definity for targeted blood-brain barrier disruption: a feasibility study. Ultrasound Med Biol 2007;33:584590.

84. Kinoshita M, McDannold N, Jolesz FA, Hynynen K. Targeted delivery of antibodies through the blood-brain barrier by MRIguided focused ultrasound. Biochem Biophys Res Commun 2006;340:1085-1090.

85. Etame AB, Diaz RJ, O'Reilly MA, et al. Enhanced delivery of gold nanoparticles with therapeutic potential into the brain using MRI-guided focused ultrasound. Nanomedicine 2012;8:11331142 .

86. Burgess A, Huang Y, Querbes W, Sah DW, Hynynen K. Focused ultrasound for targeted delivery of siRNA and efficient knockdown of htt expression. J Control Release 2012;163:125-129.

87. Thévenot E, Jordao JF, O'Reilly MA, et al. Targeted delivery of self-complementary adeno-associated virus serotype 9 to the brain, using magnetic resonance imaging-guided focused ultrasound. Hum Gene Ther 2012;23:1144-1155.

88. Huang Q, Deng J, Wang F, et al. Targeted gene delivery to the mouse brain by MRI-guided focused ultrasound-induced bloodbrain barrier disruption. Exp Neurol 2012;233:350-356.

89. Jordão JF, Ayala-Grosso CA, Markham K, et al. Antibodies targeted to the brain with image-guided focused ultrasound reduces amyloid- $\beta$ plaque load in the TgCRND8 mouse model of Alzheimer's disease. PLOS ONE 2010;5:e10549.

90. Fan C, Ting C, Lin H, et al. SPIO-conjugated, doxorubicin-loaded microbubbles for concurrent MRI and focused-ultrasound enhanced brain-tumor drug delivery. Biomaterials 2013;34:3706-3715.

91. Nance E, Timbie K, Miller GW, et al. Non-invasive delivery of stealth, brain-penetrating nanoparticles across the blood- brain barrier using MRI-guided focused ultrasound. J Control Release 2014;189:123-132.

92. Åslund AK, Berg S, Hak S, et al. Nanoparticle delivery to the brain - by focused ultrasound and self-assembled nanoparticlestabilized microbubbles. J Control Release 2015;220:287-294. 
93. Lin C, Hsieh H, Pitt WG, et al. Focused ultrasound-induced bloodbrain barrier opening for non-viral, non-invasive, and targeted gene delivery. J Control Release 2015;212:1-9.

94. McDannold N, Arvanitis CD, Vykhodtseva N, Livingstone MS. Temporary disruption of the blood-brain barrier by use of ultrasound and microbubbles: safety and efficacy evaluation in rhesus macaques. Cancer Res 2012;72:3652-3663.

95. Marquet F, Tung Y, Teichert T, Ferrera VP, Konofagou EE. Noninvasive, transient and selective blood-brain barrier opening in non-human primates in vivo. PLOS ONE 2011;6:e22598.

96. Konofagou EE. Optimization of the ultrasound-induced bloodbrain barrier opening. Theranostics 2012;2:1223-1237.

97. Kinoshita M, McDannold N, Jolesz FA, Hynynen K. Noninvasive localized delivery of herceptin to the mouse brain by MRI-guided focused ultrasound-induced blood-brain barrier disruption. Proc Natl Acad Sci U S A 2006;103:11719-11723.

98. Aryal M, Park J, Vykhodtseva N, Zhang Y, McDannold N. Enhancement in blood-tumor barrier permeability and delivery of liposomal doxorubicin using focused ultrasound and microbubbles: Evaluation during tumor progression in a rat glioma model. Phys Med Biol 2015;60:2511.

99. McDannold N, Clement GT, Black P, Jolesz F, Hynynen K. Transcranial magnetic resonance imaging- guided focused ultrasound surgery of brain tumors: Initial findings in 3 patients. Neurosurgery 2010;66:323-332.

100. Wang F, Shi Y, Lu L, et al. Targeted delivery of GDNF through the blood-brain barrier by MRI-guided focused ultrasound. PLOS ONE 2012;7:e52925.

101. Samiotaki G, Acosta C, Wang S, Konofagou EE. Enhanced delivery and bioactivity of the neurturin neurotrophic factor through focused ultrasound-mediated blood-brain barrier opening in vivo. J Cereb Blood Flow Metab 2015;35:611-622.

102. Fan $\mathrm{CH}$, Ting $\mathrm{CY}$, Lin $\mathrm{CY}$, et al. Noninvasive, targeted, and nonviral ultrasound-mediated GDNF-plasmid delivery for treatment of Parkinson's disease. Sci Rep 2016;6:19579.

103. Alonso A, Reinz E, Leuchs B, et al. Focal delivery of AAV2/1transgenes into the rat brain by localized ultrasound-induced BBB opening. Mol Ther 2013;2:e73.

104. Downs ME, Buch A, Sierra C, et al. Long-term safety of repeated blood-brain barrier opening via focused ultrasound with microbubbles in non-human primates performing a cognitive task. PLOS ONE 2015;10:e125911.

105. Kobus T, Vykhodtseva N, Pilatou M, Zhang Y, McDannold N. Safety validation of repeated Blood-Brain barrier disruption using focused ultrasound. Ultrasound Med Biol 2016;42:481-492.

106. Bartus R, Kordower J, Johnson E, et al. Post-mortem assessment of the short and long-term effects of the trophic factor neurturin in patients with $\alpha$-synucleinopathies. Neurobiol Dis 2015;78:162-171.

107. Warren Olanow C, Bartus RT, Baumann TL, et al. Gene delivery of neurturin to putamen and substantia nigra in Parkinson disease: a double-blind, randomized, controlled trial. Ann Neurol 2015;78:248-257.

108. Allen PJ, Feigin A. Gene-based therapies in Parkinson's disease. Neurotherapeutics 2014;11:60-67.

109. Palfi S, Gurruchaga JM, Ralph GS, et al. Long-term safety and tolerability of ProSavin, a lentiviral vector-based gene therapy for Parkinson's disease: a dose escalation, open-label, phase $1 / 2$ trial. Lancet 2014;383:1138-1146.

110. Choi JJ, Wang S, Brown TR, Small SA, Duff KE, Konofagou EE. Noninvasive and transient blood-brain barrier opening in the hippocampus of Alzheimer's double transgenic mice using focused ultrasound. Ultrason Imaging 2008;30:189-200.

111. Burgess A, Dubey S, Yeung S, et al. Alzheimer disease in a mouse model: MR imaging-guided focused ultrasound targeted to the hippocampus opens the blood-brain barrier and improves pathologic abnormalities and behavior. Radiology 2014;273:736-745.
112. Jordão JF, Thévenot E, Markham-Coultes K, et al. Amyloid- $\beta$ plaque reduction, endogenous antibody delivery and glial activation by brain-targeted, transcranial focused ultrasound. Exp Neurol 2013;248:16-29.

113. Leinenga G, Gotz J. Scanning ultrasound removes amyloid-beta and restores memory in an Alzheimer's disease mouse model. Sci Transl Med 2015;7:278ra33.

114. Scarcelli T, Jordão JF, O'Reilly MA, Ellens N, Hynynen K, Aubert I. Stimulation of hippocampal neurogenesis by transcranial focused ultrasound and microbubbles in adult mice. Brain Stimul 2014;7:304-307.

115. Arbab AS, Jordan EK, Wilson LB, Yocum GT, Lewis BK, Frank JA. In vivo trafficking and targeted delivery of magnetically labeled stem cells. Hum Gene Ther 2004;15:351-360.

116. Shen WB, Plachez C, Chan A, et al. Human neural progenitor cells retain viability, phenotype, proliferation, and lineage differentiation when labeled with a novel iron oxide nanoparticle, molday ION rhodamine B. Int J Nanomedicine 2013;8:4593-4600.

117. Shen W, Plachez C, Tsymbalyuk O, et al. Cell-based therapy in TBI: Magnetic retention of neural stem cells in vivo. Cell Transplant 2016;25:1085-1099.

118. Fishman P, Shen W, Yarowsky P, Nguyen B, Frenkel V. Enhancement of FUS mediated delivery of stem cells to the brain. Abstracts of the Fifth International Symposium on Focused Ultrasound August 2016. Available at: https://www.xcdsystem. com/fus2016/program/.

119. de Munter JP, Melamed E, Wolters EC. Stem cell grafting in parkinsonism-why, how and when. Parkinsonism Relat Disord 2014;20:S150-S153.

120. Daadi MM, Grueter BA, Malenka RC, Redmond Jr DE, Steinberg GK. Dopaminergic neurons from midbrain-specified human embryonic stem cell-derived neural stem cells engrafted in a monkey model of parkinson's disease. PLOS ONE 2012;7:e41120.

121. Emborg ME, Zhang Z, Joers V, et al. Intracerebral transplantation of differentiated human embryonic stem cells to hemiparkinsonian monkeys. Cell Transplant 2013;22:831-838.

122. Silvestrini MT, Yin D, Coppes VG, et al. Radially branched deployment for more efficient cell transplantation at the scale of the human brain. Stereotact Funct Neurosurg 2013;91:92-103.

123. Mano Y, Saito R, Haga Y, et al. Intraparenchymal ultrasound application and improved distribution of infusate with convectionenhanced delivery in rodent and nonhuman primate brain. $J$ Neurosurg 2016;124:1490-1500.

124. Wang S, Karakatsani ME, Fung C, Sun T, Acosta C, Konofagou E. Direct brain infusion can be enhanced with focused ultrasound and microbubbles. J Cereb Blood Flow Metab 2017;37:706-714.

125. Hersh DS, Nguyen BA, Dancy JG, et al. Pulsed ultrasound expands the extracellular and perivascular spaces of the brain. Brain Res 2016;1646:543-550.

126. Chen H, Chen CC, Acosta C, Wu S, Sun T, Konofagou EE. A new brain drug delivery strategy: Focused ultrasound-enhanced intranasal drug delivery. PLOS ONE 2014;9:e108880.

127. McDannold N, Zhang YZ, Power C, Jolesz F, Vykhodtseva N. Nonthermal ablation with microbubble-enhanced focused ultrasound close to the optic tract without affecting nerve function. $J$ Neurosurg 2013;119:1208-1220.

128. Monteith S, Snell J, Eames M, Kassell NF, Kelly E, Gwinn R. Transcranial magnetic resonance-guided focused ultrasound for temporal lobe epilepsy: a laboratory feasibility study. $J$ Neurosurg 2016;125:1557-1564.

129. King RL, Brown JR, Pauly KB. Localization of ultrasound-induced in vivo neurostimulation in the mouse model. Ultrasound Med Biol 2014:40:1512-1522.

130. Yoo SS, Kim H, Min BK, Franck E, Park S. Transcranial focused ultrasound to the thalamus alters anesthesia time in rats. Neuroreport 2011;22:783-787. 
131. Tufail Y, Matyushov A, Baldwin N, et al. Transcranial pulsed ultrasound stimulates intact brain circuits. Neuron 2010;66: 681-694.

132. Krasovitski B, Frenkel V, Shoham S, Kimmel E. Intramembrane cavitation as a unifying mechanism for ultrasound-induced bioeffects. Proc Natl Acad Sci U S A 2011;108:3258-3263.

133. Plaksin M, Kimmel E, Shoham S. Cell-type-selective effects of intramembrane cavitation as a unifying theoretical framework for ultrasonic neuromodulation. eNeuro 2016;3(3). pii: ENEURO.013615.2016.

134. Legon W, Sato TF, Opitz A, et al. Transcranial focused ultrasound modulates the activity of primary somatosensory cortex in humans. Nat Neurosci 2014;17:322-329.

135. Mueller J, Legon W, Opitz A, Sato TF, Tyler WJ. Transcranial focused ultrasound modulates intrinsic and evoked EEG dynamics. Brain Stimul 2014;7:900-908. 